\title{
A Systematic Review of Dog-Assisted Therapy in Children with Behavioural and Developmental Disorders
}

\author{
Candela J. Hüsgen ${ }^{1,2} \cdot$ Nienke C. Peters-Scheffer ${ }^{1,3} \cdot$ Robert Didden $^{1,4}$
}

Accepted: 28 December 2021 / Published online: 14 January 2022

(c) The Author(s) 2022

\begin{abstract}
Objectives Animal-assisted therapy with dogs is regularly used in children with behavioural and developmental disorders. Aims of this systematic review were threefold: to analyse the methodological quality of studies on dog-assisted therapy (DAT) for children with behavioural and developmental disorders, to determine to which extent the studies on DAT adhere to the quality criteria developed by the International Association of Human Animal Interaction Organisation (IAHAIO) and to describe the characteristics of the participants, the intervention and the outcomes.

Method Three databases (i.e. PsycInfo, MedLine and Eric) were searched, and 14 studies on DAT were included. The Joanna Briggs Institute checklist (JBIC) and the quality criteria developed by the IAHAIO were used during data extraction. Characteristics of the participants, the intervention, the therapy dogs and the outcomes of the studies were summarised.

Results Six of the 14 included studies reported significant outcomes of DAT, whereof six in the social domain and two in the psychological domain. However, scores on the JBIC indicated low to moderate methodological quality and only three of the included studies adhered to the IAHAIO quality criteria.

Conclusions DAT is a promising intervention for children with behavioural and developmental disorders, especially for children with autism spectrum disorder. A clear description of the therapy's components, the role of the therapy dog and analysis of the treatment integrity and procedural fidelity would improve the methodological quality of the studies and the field of dog-assisted interventions.
\end{abstract}

Keywords Animal-assisted therapy · Dog-assisted therapy · Children · Behavioural and developmental disorders $\cdot$ Review

The growing body of literature indicates that animal-assisted therapy (AAT) with dogs is a promising intervention for children with different types of behavioural and developmental disorders (Hoagwood et al., 2016; Schuck et al., 2018). In AAT, an animal is present during a therapeutic

Candela J. Hüsgen

candela.huesgen@ru.nl

Nienke C. Peters-Scheffer

nienke.peters-scheffer@ru.nl

Robert Didden

robert.didden@ru.nl

1 Behavioural Science Institute, Radboud University, PO Box 9104, 6500 HE Nijmegen, The Netherlands

2 Hulphond, PO Box 24, 5373 ZG Herpen, The Netherlands

3 Driestroom, Industrieweg-Oost 27, 6662 NE Elst, The Netherlands

4 Trajectum, Hanzeallee 2, 8017 KZ Zwolle, The Netherlands intervention together with the client and the therapist, and it is used to improve the social skills of the client, to reduce psychological symptoms and/or to promote neurobiological processes (Gut et al., 2018; Jegatheesan et al., 2018). Given their ability to adapt to human behaviour, read and react to human (body) language, availability and trainability, dogs are one of the most common animals used in AAT (Duranton \& Gaunet, 2018; Glenk, 2017; Miklósi \& Topál, 2013). In a recent study from Kerulo et al. (2020), the perspective of 239 professionals working in the field of animal-assisted interventions (AAI) was investigated, and it was found that most professionals (88\%) were working with dogs.

During therapeutic interventions, dogs can be integrated in an active or passive way. In a study by Hill et al., (2019a, 2019b), dogs were integrated in either an active or a passive way depending on the needs and goals of the client. For example, children diagnosed with autism spectrum disorder were working actively with the dog, playing catch to strengthen the independent performance of the child, while 
other children were working passively with the dog, learning how to recognise emotions. Also, passive AAT programmes for children to enhance their reading skills are commonly used in libraries and schools (Henderson et al., 2020). While the child is reading, the dog is sitting or lying next to the child without further interaction. Another distinction often made in AAT is that the dogs are integrated into individual therapy or group therapy. For example, Wohlfarth et al. (2013) investigated the motivational effects of the active use of dogs in groupwise training for obese children, while Griffioen et al. (2019) studied the changes in synchronicity between children and dogs during individual therapy sessions. A further distinction can be found in the setup of the therapy session. In some studies, AAT is provided by a therapist who is responsible for both providing therapy to the client and handling the dog (e.g. Wijker et al., 2019). In other studies, a therapist provides the therapy but is assisted by a dog handler who is responsible for ensuring the welfare and safety of the dog (e.g. Flynn et al., 2018; Hediger et al., 2019). Also, training and certification of the therapist, handlers and dogs differed across studies.

During the past decade, several attempts have been made to summarise the effectiveness of AAT in children and adults, including children with autism spectrum disorder (Fung \& Leung, 2014), adults with autism spectrum disorder (Wijker et al., 2021), children with pervasive developmental disorder (Martin and Farnum, 2002), children with Down syndrome (Griffioen et al., 2019), children who were sexually abused (Dietz et al., 2012) and children with attention-deficit hyperactivity disorder (Juríčková, et al., 2020). Beetz et al. (2021) categorised the outcomes of DAT as (1) improvements in social skills such as empathy and communication (Hill et al., 2019a, 2019b; Stevenson et al., 2015; Wells et al., 2019), (2) psychological effects such as improvements in concentration and motivation (Busch et al., 2016; Hill et al., 2019a, 2019b; Schuck et al., 2013, 2018) and (3) neurobiological effects such as decreases in heart rate and blood pressure (Nagasawa et al., 2015). While most studies on AAT focussed on the effectiveness of dogassisted therapy (DAT), studies systematically investigating the therapy approaches, settings and research methodology are scarce (Fine et al., 2019).

In line with the rapid increase of AAT providers, several organisations, including Pets Partners, the International Association of Human Animal Interaction Organisation (IAHAIO), Animal Assisted Intervention International and the International Society for Animal Assisted Therapy, were founded to protect and ensure the welfare and wellbeing of the clients, therapists and animals participating in AAT. These organisations have introduced several guidelines for healthcare interventions, including the role and wellbeing of animals. Although these guidelines are regularly used in clinical practice, little is known about how these are applied in current and past AAT studies. Therefore, the aim of this review is threefold. First, we use the Joanna Briggs Institute checklist (JBIC) to analyse the methodological quality of the studies evaluating DAT in children with behavioural and developmental disorders. Second, we investigate to which extent studies on DAT adhere to the AAT quality criteria developed by the IAHAIO. And third, we summarise the characteristics of the participants, intervention, therapy dogs and outcomes of the studies.

\section{Method}

\section{Procedure}

Three databases (i.e. PsycInfo, MedLine and Eric) were searched, combining standard terms used in children with behavioural and developmental disorders and AAT with dogs to identify studies (see Fig. 1 for the selection process). Two academic librarian were consulted to develop the following search strings: (Child* or adolescent* or infant* or toddler* or student* or pre-schooler* or puber*) AND (therap* or intervention* or coach* or activit* or consult* or training or education or learning or treatment* AND ( $\mathrm{dog}^{*}$ assist* or assis* $\operatorname{dog}^{*}$ or $\operatorname{dog}^{*}$ based or canis or kanis or (therap* adj3 dog*)) OR (animal* assist* or assist* animal* or animal* based or animal*) OR (trained dog* or therapy dog* or service $\operatorname{dog} *)$ OR (dog* assist* or assis* dog* or dog* based or canis or kanis or (therap* adj3 dog*)). The search was limited to include only studies published between 2008 and 2020 in peer-reviewed journals and published in English (i.e. 6 years before and 6 years after the publication of the IAHAIO guidelines; Jegatheesan et al., 2018). Duplications were removed, which resulted in 529 studies. Next, the first and second authors screened all titles and abstracts for the four inclusion criteria, which were based on the PICO criteria (Higgins et al., 2019): (1) participants were children between 0 and 18 years of age; (2) a dog was included in the intervention; (3) the interventions were implemented in a therapeutic setting with a healthcare professional, a therapeutic goal and multiple individual therapy sessions; and (4) effects of the interventions on the participants were measured. As pointed out by Chandler (2017) and Seivert et al. (2016), a close relationship between a therapy dog and client is an essential component of DAT. Therefore, we only evaluated individual therapy sessions in this review. Differences between the authors were discussed until consensus was reached.

After the selection process, 13 studies were identified, and the references of these 13 studies were checked for relevant studies. One study that met the inclusion criteria was identified and also included in the review. This resulted in a total of 14 studies that were analysed by the first author. 
Fig. 1 Prisma flow chart of data extraction

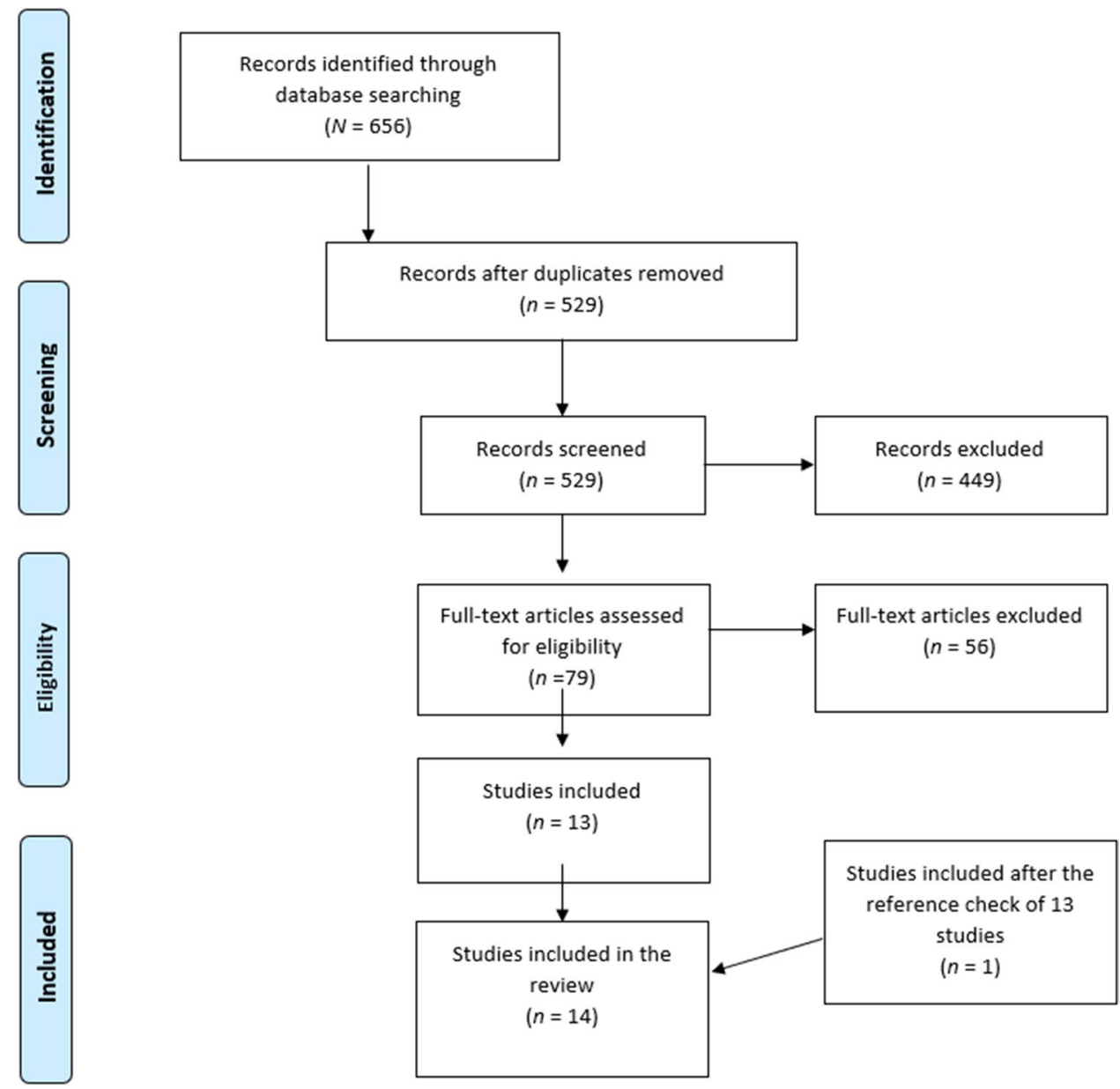

\section{Quality of the Studies}

The second author analysed three $(21 \%)$ of the included studies. Hill et al., (2018; 2019a, b) described the same research project from different perspectives; these studies were treated as one study. During the selection procedure, interrater reliability between the first and second authors was calculated, using Cohen's $K$ for evaluating the agreement between raters (Cicchetti, 1994). Substantial agreement between the first and second authors was found, $K=0.66$, $p<0.001$ (McHugh, 2012).

\section{Data Analysis}

Two forms were used during data extraction. First, the JBIC was used to analyse the quality of the studies according to the current standard for intervention studies. Second, a customised form based on the PRISMA extraction form and IAHAIO Whitepaper was used to evaluate to which extent studies adhered to the quality guidelines of the IAHAIO and to describe the outcomes of the studies. The first author extracted the data of all studies. In addition, the second author analysed three of the included studies to calculate interrater agreement. Substantial agreement was found, $K=0.61, p<0.001$ (McHugh, 2012).
The Joanna Brigg Institute checklists (JBIC) for Case Studies (Moola et al., 2020), Qualitative Research (Lockwood et al., 2015), Quasi-Experimental Studies and Randomized Controlled Trials (Tufanaru et al., 2020) were used to evaluate the quality of the study relative to research standards for interventions studies. The JBIC contains different checklists, which measure the methodical quality and risks of bias of the studies (Ma et al., 2020; Xiao et al., 2019). Depending on the study format, the matching checklist of the JBIC was used. By answering 8 to 13 closed-ended questions (depending on the type of study design reviewed by the checklist), the JBIC evaluate the quality of the study design, completeness of the information on the participants and research methods used. The JBIC were chosen because of their availability for all four types of studies covered in the present review and its up-to-dateness. The results are presented in percentages of completeness based on the number of criteria with a range from 0 (information is not described) to 100 (all information is available and described). 


\section{AAT Quality Criteria and Outcome}

A customised data extraction form was used to extract data related to the IAHAIO criteria and outcomes of the studies. The form was based on the PRISMA checklist and the IAHAIO Whitepaper (Jegatheesan et al., 2018). The PRISMA was chosen to ensure complete and transparent information extraction (Liberati et al., 2009), while the IAHAIO Whitepaper was used to operationalise AAT and as a guideline for the adequate handling of human and dog welfare during the interventions. The form consisted of three components: (1) characteristics of the participants (i.e. gender, age and diagnosis), (2) characteristics of the dog (i.e. breed, gender, age, colour, neutered/pregnant, owner) and (3) outcome variables (see Table 1).

\section{Results}

\section{The Current Standard for Intervention Studies}

Of all 14 studies, 9 used a quasi-experiment design, and 3 used a randomised control trial design. Also, 1 case study and 1 qualitative study were included. The qualitative study had the highest quality score on the JBIC, with $90 \%$ of the criteria completed. The 3 randomised controlled trials scored on average 74\% (range: 69-77\%). The studies that used a quasi-experimental design $(n=9)$ had a mean score of $62 \%$ (range: $44-78 \%$ ), and the case study had the lowest score with $50 \%$ of the criteria completed on the JBIC. According to the JBIC criteria, most studies reported sufficient information about the participants. However, only 6 studies used a control group. Most control groups received a similar treatment as the experimental group but without a dog, making blinded rating difficult. The JBIC scores of the individual studies can be found in Table 2 .

\section{AAT Quality Criteria}

To assess to which extent the studies adhered to the AAT quality criteria (Jegatheesan et al., 2018), the characteristics of participants, dogs, professionals and intervention were summarised first.

The number of participants ranged from 1 to 117 . It included children diagnosed with autism spectrum disorder (11 of the 14 studies), children in paediatric care ( 2 studies), incarcerated youth (1 study) and participants with Down syndrome (2 studies). One of the latter two studies included both children with autism spectrum disorder and Down syndrome. The age of the participants ranged from 4 to 19 years, and most participants were boys (70\%). The therapeutical orientation was mentioned in 6 studies, including occupational therapy (2 studies) and applied behaviour analysis (1 study). Characteristics of the studies are summarised in Table 2 .

In the 14 studies, between one and eleven dogs were used. In line with the IAHAIO-criteria, the studies were screened on the following 6 characteristics of the dog: (1) breed, (2) gender, (3) age, (4) colour, (5) neutered/pregnant and (6) and dog-owner (see Table 2). In most studies, information regarding the characteristics of the dogs was scarce. Of the six characteristics, most studies reported fewer than three, with an average of 2.5 characteristics. In 6 studies, a triadic setting (i.e. dog, child and therapist) was used, while in 5 studies, a dog handler was added, and a quadratic setting was used (i.e. dog, dog handler, child and therapist). In three studies, this information was not reported.

In the IAHAIO Whitepaper, nine criteria are identified to protect the welfare of the participants and the dogs (Table 1). The first three criteria focussed on the welfare and background of the participants and the other six on the welfare, training and handling of the dog. All studies reported information on one or more of the first three criteria that were related to the welfare and background of the participants. Eight studies reported that participants were checked on
Table 1 Characteristics of participants and dogs and IAHAIO criteria

\begin{tabular}{ll}
\hline AAT quality criteria and outcome & \\
\hline Participant's characteristics & Age, gender and diagnosis \\
Dog's characteristics & Age, gender, breed, colour, neutered/pregnant, owner \\
IAHAIO guideline & (1) participants are not allergic to dogs; (2) professional is certified to \\
& provide therapy to children; (3) cultural background of participants \\
& is known; (4) dogs used during therapy are socialised; (5) dogs are \\
& registered therapy dogs; (6) evaluation of the dog's behaviour; (7) \\
& dogs have been checked by a veterinarian; (8) therapist or handler is \\
& certified for working with dogs; and (9) duration of therapy session \\
& is between 30 and 45 min \\
& Social, psychological, neurobiological \\
\hline
\end{tabular}

Based on IAHAIO White Paper (Jegatheesan et al. 2018) 


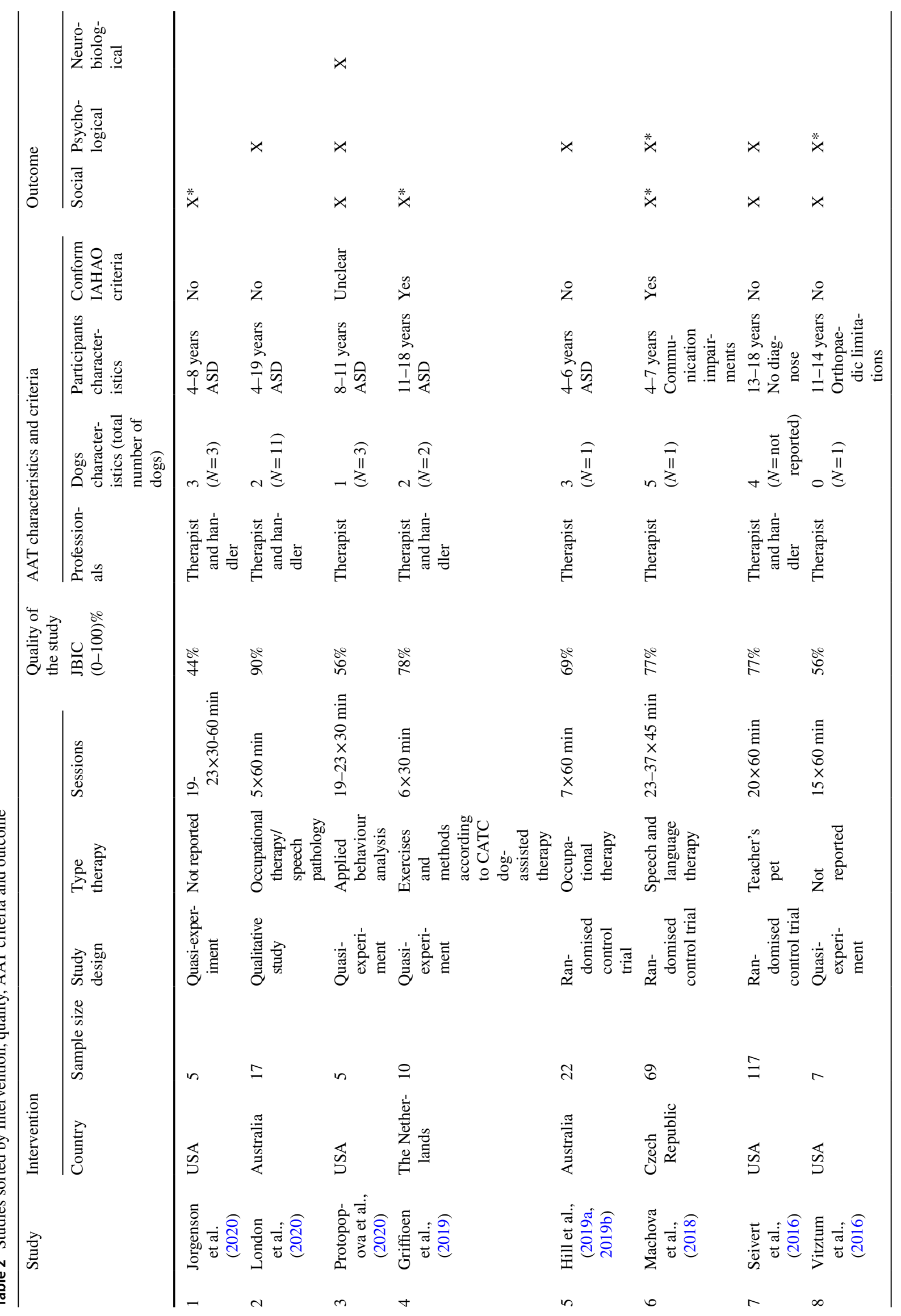




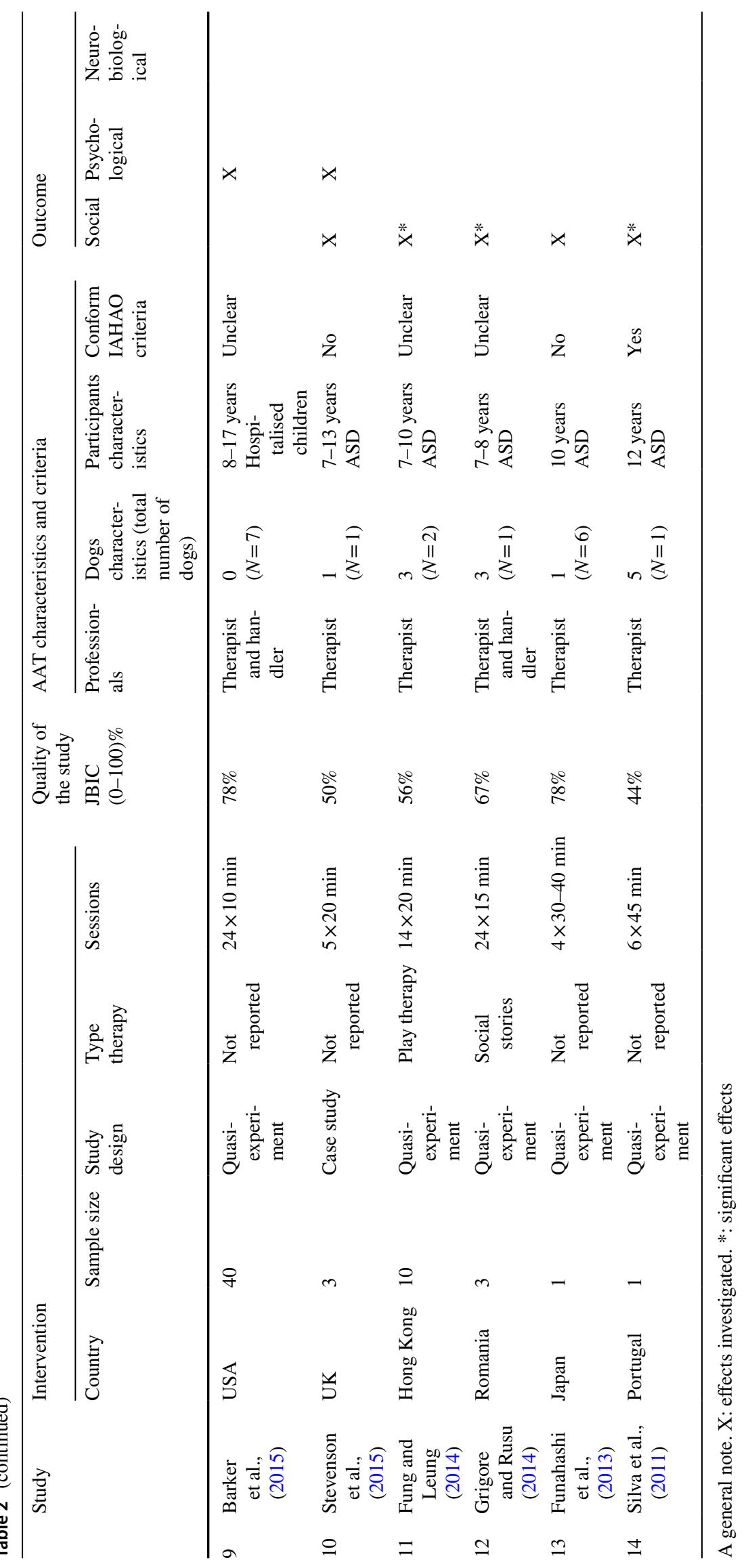


dog-related allergies, and in 12 studies, the education of the therapist who provided AAT was reported. Only one study reported the cultural background of the participants. Most studies reported scarce information on the six criteria related to the characteristics and welfare of the dog (i.e. breed, gender, age, colour, neutered/pregnant and owner). The most shared information about the therapy dogs was that they are socialised (10/14), registered as therapy dog (9/14), evaluated (7/14) and veterinarian checked (5/14). Twelve studies also reported that the therapist and/or dog handler were educated and certified in dog training and/or handling. All studies reported the duration of the sessions. However, most studies did not report breed, colour, age or gender and also five of the 14 studies described therapy sessions with a longer duration (i.e. $60 \mathrm{~min}$ ) than prescribed in the IAHAIO criteria (i.e. $30-45 \mathrm{~min}$ ).

Only three studies adhered to all AAT criteria, seven did not adhere to the criteria. Four studies reported insufficient information to assess if AAT criteria were followed. Of the three studies that adhered to the guidelines, two were published before the publication of the guidelines (Jegatheesan et al., 2018). Of the eight studies that were published after the publication of the AAT criteria, only one study was designed in line with these guidelines. Although two studies referred to the IAHAIO guideline, both studies did not meet the criteria. Only five studies reported that a treatment protocol or manual was used.

\section{The Outcome of the Studies}

To evaluate the outcomes of the studies, the data were summarised and categorised into three domains. Table 2 depicts the outcomes on the three domains of effectiveness proposed by Beetz et al. (2021): social, psychological and neurobiological. Most studies (78\%) reported outcomes on the social domain, including increased social and communication skills and reduction in aggressive behaviours. Ten studies (71\%) reported psychological outcomes, including more motivation and concentration. Only one study evaluated the neurobiological outcomes of DAT and found reductions in salivary cortisol levels in five children (Protopopova et al., 2020). Six of the 14 studies (43\%) reported significant outcomes of DAT, whereof six in the social domain and two in the psychological domain. Most studies also reported anecdotal outcomes such as improved motivation or reduction of aggressive behaviour.

\section{Discussion}

This systematic review aimed to evaluate the methodological quality of studies investigating DAT in children with behavioural and developmental disorders and the extent to which these studies adhered to quality guidelines of IAHAIO
(Jegatheesan et al., 2018). Also, characteristics of participants, intervention, therapy dogs and outcomes were summarised. The quality of the 14 included studies was evaluated using two checklists. The overall methodological quality of the studies was evaluated using the JBIC, and the criteria of the IAHAIO were used to assess if studies adhered to the guidelines for human and animal welfare in AAT. Forty-four to $90 \%$ of the criteria of the JBIC were met, which is in line with other studies that assessed the methodological quality with the JBIC. For example, Kanninen et al., (2021) evaluated interventions for strengthening professionals' governance in nursing in hospitals and found that 55 to $89 \%$ of the criteria were met. Xiao et al. (2019) reviewed studies on the effect of therapy on dignity, psychological wellbeing and quality of life among cancer patients. With 62 to $89 \%$ of the criteria met, none of the studies included in their review fulfilled all JBIC criteria (Xiao et al., 2019). JBIC scores of the 14 included studies indicated low to moderate methodological quality.

While in most studies all JBIC criteria regarding the participants were met, criteria regarding the randomisation and blinding of the assessors were not met. Furthermore, it was difficult to extract data related to the intervention as many studies did not report sufficient information regarding the intervention and control conditions. Often, no information regarding theories underlying the intervention, the treatment protocol, and intervention techniques was given, and treatment integrity and procedural fidelity were often not measured. Included studies were characterised by a high degree of heterogeneity regarding the participants, the intervention and the control conditions used. Although all studies investigated DAT, the number of sessions varied from 4 to 37 and duration of the sessions varied between 5 and $60 \mathrm{~min}$. Also, the therapeutic function and tasks of the therapy dog differed across studies. While in the study of Seivert et al. (2016) the interaction between the dog and the child was integrated into the therapy programme, Protopopova et al. (2020) used the therapy dog as a reinforcer for the participation of the child in an educational task. Most studies provided little information on the treatment and the procedures related to the dog's role in the treatment and setting of the treatment. No study measured the long-term effects of DAT.

The IAHAIO is the global association of organisations that engage in AAI. Their criteria for AAI outline the best practices in delivering DAT to ensure the health and wellbeing of both clients and therapy dogs. As these criteria are seen as the golden standard in clinical practice, they were used to evaluate the quality of DAT provided in the included studies. As no clear definition, description and protocol of DAT exist, recommendations of the IAHAIO were summarised based on nine criteria to evaluate the quality of the intervention. Unfortunately, interventions often did not adhere to these criteria or information related to these criteria was lacking, making it impossible to determine if studies 
adhered to the criteria. For example, the criteria state that sessions should be time-limited (30-45 $\mathrm{min}$ ) to prevent the dogs from becoming overworked or overwhelmed. However, five of the 14 studies reported sessions longer than 45 min (Hill et al., 2019a, 2019b; Jorgenson et al., 2020; London et al., 2020; Seivert et al., 2016; Vitztum et al., 2016). In addition, AAI should only be performed with the assistance of animals in good health, well-socialised with humans and trained adequately with humane techniques (Jegatheesan et al., 2018). While Seivert et al. (2016) referred to the IAHAIO criteria, they used dogs from an animal shelter. These dogs had behavioural issues such as jumping and pulling and lacked socialisation. Often, characteristics of the dogs, such as their age or veterinarian checks, were not reported, making it difficult to conclude if studies adhered to the IAHAIO criteria.

The therapy dog is a crucial element in DAT. Clear protocols are needed to ensure the wellbeing and safety of clients, therapists and dogs (Jegatheesan et al., 2018) and to operationalise DAT further. However, little information on the dogs was provided in the current studies. Only some studies provided some information about the dog's characteristics, the dog's role during the therapy session and the treatment location and materials. For example, the dog can be used to reinforce the social behaviour of a child instead of using edible and/or social reinforcers provided by the therapist (Protopopova et al., 2020). However, the dog can also participate and interact with the child during a task or exercise (Hill et al., 2019a, 2019b). While characteristics of the dogs, such as breed and colour, might influence the effectiveness of DAT, most studies lacked data on these characteristics. For example, colour differences might influence the outcomes of DAT. Lavan and Knesl (2015) showed that black dogs remained longer in shelters than dogs with other colours because black dogs look more menacing and are more difficult to read during interactions. It is essential to report these characteristics to compare outcomes between studies and learn more about the effect in DAT.

Seven of the 14 studies identified significant effects of DAT. Furthermore, in the qualitative study, parents and therapists reported that children were more engaged, showed more enjoyment, and were more motivated in the therapy dog's presence (London et al., 2020). In addition to the quantitative outcomes, many studies reported anecdotal outcomes, such as increased motivation and decreased aggressive behaviour (London et al., 2020). Since most of these studies did not measure increased motivation and decreased aggressive behaviour, future studies should investigate these effects using valid and reliable measures and study designs.

\section{Limitations and future research}

Although the findings of the present review show that DAT is a promising intervention for children with behavioural and developmental disorders, the results also emphasise that more robust research methods are warranted to strengthen the current knowledge base. To further advance the field, future studies must include a clear description of the treatment components and the role of the therapy dog in the intervention. Also, the context wherein DAT is provided should be clearly described to enhance the replicability of the results. Therefore, clear guidelines need to be developed that describe the characteristics of participants, therapy dogs and interventions that should be reported in interventions studies with animals. As treatment integrity and procedural fidelity of DAT were often not assessed and different outcome measures were used, it is not easy to compare and generalise the results between studies. Future studies should include measures on treatment integrity and procedural fidelity and measures to evaluate the short-term and long-term effectiveness of DAT. As several studies reported anecdotal outcomes such as improved motivation or reduction of aggressive behaviour, future studies should include measures on these outcomes. Furthermore, qualitative studies should analyse the experiences reported by therapists and children, while observational study designs could be employed to get more insight into the underlying theories and working mechanisms of DAT. These findings may be limited because the information needed to analyse the IAHAIO criteria was lacking in some studies.

Acknowledgements This study was funded by Stichting Hulphond Nederland.

Author Contribution C. J. H.: designed the study, conducted the literature search, analysed the studies, and wrote the manuscript. N. P. S.: assisted in the data analyses, and collaborated in designing and writing the study. R. D.: collaborated in designing, writing, and editing the paper. All authors approved the final version of the manuscript before submission.

\section{Declarations}

Conflict of Interest The authors declare no competing interests.

Open Access This article is licensed under a Creative Commons Attribution 4.0 International License, which permits use, sharing, adaptation, distribution and reproduction in any medium or format, as long as you give appropriate credit to the original author(s) and the source, provide a link to the Creative Commons licence, and indicate if changes were made. The images or other third party material in this article are included in the article's Creative Commons licence, unless indicated otherwise in a credit line to the material. If material is not included in the article's Creative Commons licence and your intended use is not permitted by statutory regulation or exceeds the permitted use, you will need to obtain permission directly from the copyright holder. To view a copy of this licence, visit http://creativecommons.org/licenses/by/4.0/.

\section{References}

Barker, S. B., Knisely, J. S., Schubert, C. M., Green, J. D., \& Ameringer, S. (2015). The effect of an animal-assisted intervention on anxiety and pain in hospitalized children. Anthrozoös, 28(1), 101-112. https://doi.org/10.2752/089279315x14129350722091 
Beetz, A., Wohlfarth, R., \& Kotraschal, K. (2021). Tiergestützt intervention [Animal assisted intervention] (Vol. 2). Ernst Reinhardt $\mathrm{GmbH} \& \mathrm{Co}$.

Busch, C., Tucha, L., Talarovicova, A., Fuermaier, A. B. M., LewisEvans, B., \& Tucha, O. (2016). Animal-assisted interventions for children with attention deficit/hyperactivity disorder. Psychological Reports, 118(1), 292-331. https://doi.org/10.1177/00332 94115626633

Chandler, C. K. (2017). Animal-assisted therapy in counseling (3rd ed.). Routledge.

Cicchetti, D. V. (1994). Guidelines, criteria, and rules of thumb for evaluating normed and standardized assessment instruments in psychology. Psychological Assessment, 6, 284-290. https://doi. org/10.1037/1040-3590.6.4.284

Dietz, T. J., Davis, D., \& Pennings, J. (2012). Evaluating animalassisted therapy in group treatment for child sexual abuse. Journal of Child Sexual Abuse, 21(6), 665-683. https://doi.org/10.1080/ 10538712.2012 .726700

Duranton, C., \& Gaunet, F. (2018). Behavioural synchronization and affiliation: Dogs exhibit human-like skills. Learning and Behavior, 46(4), 364-373. https://doi.org/10.3758/s13420-018-0323-4

Fine, A. H., Beck, A. M., \& Ng, Z. (2019). The State of animal-assisted interventions: Addressing the contemporary issues that will shape the future. International Journal of Environmental Research and Public Health, 16(20), 3997. https://doi.org/10.3390/ijerph1620 3997

Flynn, E., Roguski, J., Wolf, J., Trujillo, K., Tedeschi, P., \& Morris, K. N. (2018). A randomized controlled trial of animal-assisted therapy as an adjunct to intensive family preservation services. Child Maltreatment, 24(2), 161-168. https://doi.org/10.1177/ 1077559518817678

Funahashi, A., Gruebler, A., Aoki, T., Kadone, H., \& Suzuki, K. (2013). The smiles of a child with autism spectrum disorder during an animal-assisted activity may facilitate social positive behaviors: Quantitative analysis with smile-detecting interface. Journal of Autism and Developmental Disorders, 44(3), 685-693. https://doi.org/10.1007/s10803-013-1898-4

Fung, S. C., \& Leung, A. S. M. (2014). Pilot study investigating the role of therapy dogs in facilitating social interaction among children with autism. Journal of Contemporary Psychotherapy, 44(4), 253-262. https://doi.org/10.1007/s10879-014-9274-z

Glenk, L. (2017). Current perspectives on therapy dog welfare in animal-assisted interventions. Animals, 7(12), 7. https://doi.org/10. 3390/ani7020007

Griffioen, R. E., Steen, S., Verheggen, T., Enders-Slegers, M., \& Cox, R. (2019). Changes in behavioural synchrony during dog-assisted therapy for children with autism spectrum disorder and children with Down syndrome. Journal of Applied Research in Intellectual Disabilities, 33(3), 398-408. https://doi.org/10.1111/jar.12682

Grigore, A. A., \& Rusu, A. S. (2014). Interaction with a therapy dog enhances the effects of social story method in autistic children. Society \& Animals, 22(3), 241-261. https://doi.org/10.1163/15685 306-12341326

Gut, W., Crump, L., Zinsstag, J., Hattendorf, J., \& Hediger, K. (2018). The effect of human interaction on guinea pig behaviour in animal-assisted therapy. Journal of Veterinary Behavior, 25, 56-64. https://doi.org/10.1016/j.jveb.2018.02.004

Hediger, K., Thommen, S., Wagner, C., Gaab, J., \& Hund-Georgiadis, M. (2019). Effects of animal-assisted therapy on social behaviour in patients with acquired brain injury: A randomized controlled trial. Scientific Reports, 9(1), 5831. https://doi.org/10.1038/ s41598-019-42280-0

Henderson, L., Grové, C., Lee, F., Trainer, L., Schena, H., \& Prentice, M. (2020). An evaluation of a dog-assisted reading program to support student wellbeing in primary school. Children and Youth
Services Review, 118, 105449. https://doi.org/10.1016/j.child youth.2020.105449

Higgins, J. P. T., Thomas, J., Chandler, J., Cumpston, M., Li, T., Page, M. J., \& Welch, V. A. (2019). Cochrane handbook for systematic reviews of interventions (Wiley Cochrane Series) (2nd ed.). Wiley-Blackwell.

Hill, J., Ziviani, J., Cawdell-Smith, J., \& Driscoll, C. (2019a). Canine assisted occupational therapy: Protocol of a pilot randomised control trial for children on the autism spectrum. Open Journal of Pediatrics, 9(3), 199-217. https://doi.org/10.4236/ojped.2019. 93020

Hill, J., Ziviani, J., Driscoll, C., \& Cawdell-Smith, J. (2018). Can canine-assisted interventions affect the social behaviours of children on the autism spectrum? A systematic review. Review Journal of Autism and Developmental Disorders, 6(1), 13-25. https:// doi.org/10.1007/s40489-018-0151-7

Hill, J., Ziviani, J., Driscoll, C., \& Cawdell-Smith, J. (2019b). Canineassisted occupational therapy for children on the autism spectrum: Challenges in practice. British Journal of Occupational Therapy, 83(4), 215-219. https://doi.org/10.1177/0308022619858851

Hoagwood, K. E., Acri, M., Morrissey, M., \& Peth-Pierce, R. (2016). Animal-assisted therapies for youth with or at risk for mental health problems: A systematic review. Applied Developmental Science, 21(1), 1-13. https://doi.org/10.1080/10888691.2015. 1134267

Jegatheesan, B., Beetz, A., Ormerod, E., Johnson, R., Fine, F., Yamazaki, K., Dudzik, C., Garcia, R. M., Winkle, M., \& Choi, G. (2018). Definitions for animal assisted intervention and guidelines for wellness of animals involved [White paper]. International association of human-animal interaction organizations. https://iahaio. org/best-practice/white-paper-on-animal-assisted-interventions/

Jorgenson, C. D., Clay, C. J., \& Kahng, S. (2020). Evaluating preference for and reinforcing efficacy of a therapy dog to increase verbal statements. Journal of Applied Behavior Analysis, 53(3), 1419-1431. https://doi.org/10.1002/jaba.668.

Juríčková, V., Bozděchová, A., Machová, K., \& Vadroňová, M. (2020). Effect of animal assisted education with a dog within children with ADHD in the classroom: A case study. Child and Adolescent Social Work Journal, 37(6), 677-684. https://doi.org/10.1007/ s10560-020-00716-x

Kanninen, T., Häggman-Laitila, A., Tervo-Heikkinen, T., \& Kvist, T. (2021). An integrative review on interventions for strengthening professional governance in nursing. Journal of Nursing Management, 29(6), 1398-1409. https://doi.org/10.1111/jonm.13377

Kerulo, G., Kargas, N., Mills, D. S., Law, G., VanFleet, R., Faa-Thompson, T., \& Winkle, M. Y. (2020). Animal-assisted interventions: Relationship between standards and qualifications. People and Animals: The International Journal of Research and Practice, 3(1), 4.

Lavan, R., \& Knesl, O. (2015). Prevalence of canine infectious respiratory pathogens in asymptomatic dogs presented at US animal shelters. Journal of Small Animal Practice, 56(9), 572-576. https:// doi.org/10.1111/jsap.12389

Liberati, A., Altman, D. G., Tetzlaff, J., Mulrow, C., Gøtzsche, P. C., Ioannidis, J. P., Clarke, M., Devereaux, P., Kleijnen, J., \& Moher, D. (2009). The PRISMA statement for reporting systematic reviews and meta-analyses of studies that evaluate health care interventions: Explanation and elaboration. Journal of Clinical Epidemiology, 62(10), e1-e34. https://doi.org/10.1016/j.jclinepi. 2009.06.006

Lockwood, C., Munn, Z., \& Porritt, K. (2015). Qualitative research synthesis: Methodological guidance for systematic reviewers utilizing meta-aggregation. International Journal of Evidence-Based Healthcare, 13, 179-187.

London, M. D., Mackenzie, L., Lovarini, M., Dickson, C., \& AlvarezCampos, A. (2020). Animal assisted therapy for children and 
adolescents with autism spectrum disorder: Parent perspectives. Journal of Autism and Developmental Disorders, 50(12), 44924503. https://doi.org/10.1007/s10803-020-04512-5

Ma, L. L., Wang, Y. Y., Yang, Z. H., Huang, D., Weng, H., \& Zeng, X. T. (2020). Methodological quality (risk of bias) assessment tools for primary and secondary medical studies: What are they and which is better? Military Medical Research, 7(1), 1-11. https:// doi.org/10.1186/s40779-020-00238-8

Machová, K., Kejdanová, P., Bajtlerová, I., Procházková, R., Svobodová, I., \& Mezian, K. (2018). Canine-assisted speech therapy for children with communication impairments: A randomized controlled trial. Anthrozoös, 31(5), 587-598. https://doi.org/10. $1080 / 08927936.2018 .1505339$

Martin, F., \& Farnum, J. (2002). Animal-Assisted therapy for children with pervasive developmental disorders. Western Journal of Nursing Research, 24(6), 657-670. https://doi.org/10.1177/01939 4502320555403

McHugh, M. L. (2012). Interrater reliability: The kappa statistic. Biochemia Medica, 22(3), 276-282. https://doi.org/10.11613/bm. 2012.031

Miklósi, D., \& Topál, J. (2013). What does it take to become 'best friends'? Evolutionary changes in canine social competence. Trends in Cognitive Sciences, 17(6), 287-294. https://doi.org/10. 1016/j.tics.2013.04.005

Moola, S., Munn, Z., Tufanaru, C., Aromataris, E., Sears, K., Sfetcu, R., Currie, M., Qureshi, R., Mattis, P., Lisy, K., \& Mu, P. F. (2020). Systematic reviews of etiology and risk. In E. Aromataris \& Z. Munn (Eds.), JBI Manual for Evidence Synthesis. Available from https://synthesismanual.jbi.globalgraag

Nagasawa, M., Mitsui, S., En, S., Ohtani, N., Ohta, M., Sakuma, Y., Onaka, T., Mogi, K., \& Kikusui, T. (2015). Oxytocingaze positive loop and the coevolution of human-dog bonds. Science, 348(6232), 333-336. https://doi.org/10.1126/scien ce. 1261022

Protopopova, A., Matter, A. L., Harris, B. N., Wiskow, K. M., \& Donaldson, J. M. (2020). Comparison of contingent and noncontingent access to therapy dogs during academic tasks in children with autism spectrum disorder. Journal of Applied Behavior Analysis, 53(2), 811-834. https://doi.org/10.1002/jaba.619

Schuck, S. E. B., Emmerson, N. A., Fine, A. H., \& Lakes, K. D. (2013). Canine-assisted therapy for children with ADHD. Journal of Attention Disorders, 19, 125-137. https://doi.org/10.1177/10870 54713502080

Schuck, S. E. B., Johnson, H. L., Abdullah, M. M., Stehli, A., Fine, A. H., \& Lakes, K. D. (2018). The role of animal assisted intervention on improving self-esteem in children with attention deficit/ hyperactivity disorder. Frontiers in Pediatrics, 6, 300. https://doi. org/10.3389/fped.2018.00300
Seivert, N. P., Cano, A., Casey, R. J., Johnson, A., \& May, D. K. (2016). Animal assisted therapy for incarcerated youth: A randomized controlled trial. Applied Developmental Science, 22(2), 139-153. https://doi.org/10.1080/10888691.2016.1234935

Silva, K., Correia, R., Lima, M., Magalhães, A., \& de Sousa, L. (2011). Can dogs prime autistic children for therapy? Evidence from a single case study. The Journal of Alternative and Complementary Medicine, 17(7), 655-659. https://doi.org/10.1089/acm.2010.0436

Stevenson, K., Jarred, S., Hinchcliffe, V., \& Roberts, K. (2015). Can a dog be used as a motivator to develop social interaction and engagement with teachers for students with autism? Support for Learning, 30(4), 341-363. https://doi.org/10.1111/1467-9604. 12105

Tufanaru, C., Munn, Z., Aromataris, E., Campbell, J., \& Hopp, L. (2020). Systematic review of effectiveness. In E. Aromataris \& Z. Munn (Eds.), JBI Manual for Evidence Synthesis. Available from https://synthesismanual.jbi.global

Vitztum, C., Kelly, P. J., \& Cheng, A. L. (2016). Hospital-based therapy dog walking for adolescents with orthopedic limitations: A pilot study. Comprehensive Child and Adolescent Nursing, 39(4), 256271. https://doi.org/10.1080/24694193.2016.1196266

Wells, A. E., Hunnikin, L. M., Ash, D. P., \& Van Goozen, S. H. M. (2019). Children with behavioural problems misinterpret the emotions and intentions of others. Journal of Abnormal Child Psychology, 48(2), 213-221. https://doi.org/10.1007/s10802-019-00594-7

Wijker, C., Kupper, N., Leontjevas, R., Spek, A., \& Enders-Slegers, M. J. (2021). The effects of animal assisted therapy on autonomic and endocrine activity in adults with autism spectrum disorder: A randomized controlled trial. General Hospital Psychiatry, 72, 36-44. https://doi.org/10.1016/j.genhosppsych.2021.05.00372:36-44

Wijker, C., Leontjevas, R., Spek, A., \& Enders-Slegers, M. J. (2019). Effects of dog assisted therapy for adults with autism spectrum disorder: An exploratory randomized controlled trial. Journal of Autism and Developmental Disorders, 50(6), 2153-2163. https:// doi.org/10.1007/s10803-019-03971-9

Wohlfarth, R., Mutschler, B., Beetz, A., Kreuser, F., \& Korsten-Reck, U. (2013). Dogs motivate obese children for physical activity: Key elements of a motivational theory of animal-assisted interventions. Frontiers in Psychology, 4(796), 1-7. https://doi.org/10. 3389/fpsyg.2013.00796

Xiao, J., Chow, K. M., Liu, Y., \& Chan, C. W. (2019). Effects of dignity therapy on dignity, psychological wellbeing, and quality of life among palliative care cancer patients: A systematic review and meta-analysis. Psycho-Oncology, 28(9), 1791-1802. https://doi. org/10.1002/pon.5162

Publisher's Note Springer Nature remains neutral with regard to jurisdictional claims in published maps and institutional affiliations. 